\title{
Mathematical Modeling of an Active-Fiber Composite Energy Harvester with Interdigitated Electrodes
}

\author{
A. Jemai, ${ }^{1}$ F. Najar, ${ }^{1}$ M. Chafra, ${ }^{1}$ and Z. Ounaies ${ }^{2}$ \\ ${ }^{1}$ Applied Mechanics and Systems Research Laboratory, Tunisia Polytechnic School, University of Carthage, \\ BP 743, 2078 La Marsa, Tunisia \\ ${ }^{2}$ Department of Mechanical and Nuclear Engineering, The Pennsylvania State University, 157B Hammond Building, \\ University Park, PA 16802, USA
}

Correspondence should be addressed to F. Najar; fehmi.najar@gmail.com

Received 27 May 2013; Accepted 21 June 2013; Published 7 May 2014

Academic Editor: Mehdi Ahmadian

Copyright @ 2014 A. Jemai et al. This is an open access article distributed under the Creative Commons Attribution License, which permits unrestricted use, distribution, and reproduction in any medium, provided the original work is properly cited.

The use of active-fiber composites (AFC) instead of traditional ceramic piezoelectric materials is motivated by flexibility and relatively high actuation capacity. Nevertheless, their energy harvesting capabilities remain low. As a first step toward the enhancement of AFC's performances, a mathematical model that accurately simulates the dynamic behavior of the AFC is proposed. In fact, most of the modeling approaches found in the literature for AFC are based on finite element methods. In this work, we use homogenization techniques to mathematically describe piezoelectric properties taking into consideration the composite structure of the AFC. We model the interdigitated electrodes as a series of capacitances and current sources linked in parallel; then we integrate these properties into the structural model of the AFC. The proposed model is incorporated into a vibration based energy harvesting system consisting of a cantilever beam on top of which an AFC patch is attached. Finally, analytical solutions of the dynamic behavior and the harvested voltage are proposed and validated with finite element simulations.

\section{Introduction}

Recently advances in smart microsensors and microelectronics contributed to the development of devices with reduced size and low energy consumption. This development encouraged researchers to innovate in the field of energy sources in order to overcome the use of batteries. Given that the technological evolutions of batteries were relatively slow during the last decade, use of piezoelectric devices as energy harvesters (EHs) has progressively attracted attention of researchers. Indeed, many studies indicate the feasibility of using piezoelectric materials as power sources at different scales [1-3].

Nevertheless, monolithic piezoelectric materials, and specifically piezoelectric ceramics such as PZT, present several integration difficulties to structures characterized by curved shapes and large displacements [4]. Furthermore, due to the brittle nature of PZT, monolithic energy harvesters cannot take advantage of relatively large stroke vibrations due to their small allowable deformations. Thus, Bent et al. [5] developed a new piezoelectric composite known as activefiber composite (AFC) which consists of PZT fibers with circular cross-section embedded into an epoxy matrix and sandwiched between two sets of interdigitated electrodes (IDE). A similar one, macrofiber composite (MFC), was also developed at NASA Langley Research Center, where PZT fiber has a square cross-section [6]. These particular designs, and thanks to the IDE, use the higher $d_{33}$ mode for actuators [7] as well as energy harvesters [4].

When used as energy generators, piezocomposites with IDE are still far from fulfilling the power consumption requirements of most portable electronics, at present. In fact, several researches showed limitation for their use for energy harvesting applications $[8,9]$. In order to improve the performance of piezocomposites used as EH or actuator, several studies were conducted to redesign AFC and MFC by studying their behavior through simulations. Unfortunately, most of the proposed models are based on FE analysis because 
of the complexity of the piezocomposite structure and the presence of IDE which complicates the electric field solution $[10,11]$. Few analytical models have been also reported on EHs using IDE. Two approaches are proposed in the literature. The first approach consists in studying a representative volume element (RVE) of the piezocomposite by benefiting from the periodicity of the material's microstructure. Den Otter [12] determined a polynomial analytic expression of the electric field and the capacitance in piezocomposite with IDEs. Later, Lloyd [13] determined an analytic solution of the electric field and potential distribution using complex analysis based on the Schwarz-Christoffel's method. Schaur and Jakoby [14] presented a more efficient and versatile semianalytical model, which yields approximate expressions for the capacitance of IDEs by integrating the electrode's thickness and the covering layer. The second approach consists in taking into account the whole microstructure of the piezocomposite. Erturk et al. [15] investigated the MFC configuration for EH by deriving a distributed-parameter electromechanical model. They assumed an empirical model of the electric field distribution in order to handle the nonuniform field. Using a quasistatic analysis, Mo et al. [16] developed an analytical model for an IDE energy harvester with monolithic piezoelectric material assuming constant and unidirectional electric field. Later, Knight et al. [17] developed an optimization of the IDE EH based on analytical modeling developed by Mo et al. [16].

In this paper, we seek a parameterized mathematical model, of an AFC microstructure, to be solved analytically in order to estimate the amount of electrical voltage that could be generated by the system when excited under harmonic transversal displacement.

\section{Homogenization of Piezoelectric Fiber Composite}

The first step toward the derivation of a mathematical model of the proposed AFC based energy harvester (EH) is to estimate the global effective physical properties of the unidirectional (1-3 periodic) piezoelectric fiber composite by homogenizing its internal microstructure in order to facilitate its integration into the equation of motion of the EH. The piezocomposite under study is formed by a transversely isotropic material (PZT-5A) embedded into an isotropic Epoxy matrix (see Table 1). Hence, the resulting composite is a transversely isotropic piezoelectric material too (Figure 1(a)).

Thus, the associated linear constitutive equations may be written as

$$
\boldsymbol{\Sigma}=\mathbf{E} \boldsymbol{\Xi},
$$

where in matrix form and using Voigt notation

$$
\begin{aligned}
& \boldsymbol{\Sigma}^{t}=\left[\begin{array}{lllllllll}
\bar{\sigma}_{1} & \bar{\sigma}_{2} & \bar{\sigma}_{3} & \bar{\sigma}_{4} & \bar{\sigma}_{5} & \bar{\sigma}_{6} & \bar{D}_{1} & \bar{D}_{2} & \bar{D}_{3}
\end{array}\right], \\
& \boldsymbol{\Xi}^{t}=\left[\begin{array}{lllllllll}
\bar{\varepsilon}_{1} & \bar{\varepsilon}_{2} & \bar{\varepsilon}_{3} & \bar{\varepsilon}_{4} & \bar{\varepsilon}_{5} & \bar{\varepsilon}_{6} & -\bar{E}_{1} & -\bar{E}_{2} & -\bar{E}_{3}
\end{array}\right]
\end{aligned}
$$

and, for the IDE configuration, the electromechanical constants matrix is given by

$$
\mathbf{E}=\left(\begin{array}{ccccccccc}
C_{11}{ }^{e} & C_{12}{ }^{e} & C_{31}{ }^{e} & 0 & 0 & 0 & 0 & 0 & e_{31}{ }^{e} \\
C_{12}{ }^{e} & C_{11}{ }^{e} & C_{31}{ }^{e} & 0 & 0 & 0 & 0 & 0 & e_{31}{ }^{e} \\
C_{31}{ }^{e} & C_{31}{ }^{e} & C_{33}{ }^{e} & 0 & 0 & 0 & 0 & 0 & e_{33}{ }^{e} \\
0 & 0 & 0 & C_{44}{ }^{e} & 0 & 0 & 0 & e_{15}{ }^{e} & 0 \\
0 & 0 & 0 & 0 & C_{44}{ }^{e} & 0 & e_{15}{ }^{e} & 0 & 0 \\
0 & 0 & 0 & 0 & 0 & C_{66}{ }^{e} & 0 & 0 & 0 \\
0 & 0 & 0 & 0 & e_{15}{ }^{e} & 0 & -\epsilon_{11}{ }^{e} & 0 & 0 \\
0 & 0 & 0 & e_{15}{ }^{e} & 0 & 0 & 0 & -\epsilon_{11}{ }^{e} & 0 \\
e_{31}{ }^{e} & e_{31}{ }^{e} & e_{33}{ }^{e} & 0 & 0 & 0 & 0 & 0 & -\epsilon_{33}{ }^{e}
\end{array}\right),
$$

where $\bar{\sigma}_{i}, \bar{D}_{i}, \bar{\varepsilon}_{i}$, and $\bar{E}_{i}$ denote average values of the homogenized material for stress, electric displacement, strain, and electric field components, respectively. $C_{i j}{ }^{e}, e_{i j}{ }^{e}$, and $\epsilon_{i j}{ }^{e}$ denote the effective coefficients of elastic stiffness and piezoelectric and permittivity components at constant strain, respectively. The mean value of an effective physical property, bar notation, is defined as $\bar{f}=(1 / V) \int_{V} f d V$.

Various approaches were proposed in the literature to calculate the homogenized parameters of a two-phase piezocomposite using analytical and/or numerical homogenization techniques $[18,19]$. In this paper, we use both techniques to calculate the effective material coefficients of the piezocomposite. The obtained values are compared to each other for validation purposes. As an analytical approach, we propose the use of the Mori-Tanaka method (MTM). On the other hand, the finite element method (FEM), with the commercial package ANSYS, will be used as a numerical approach. In fact, several studies showed that these approaches are suitable for piezocomposites with short and long fibers [19, 20].

The use of the FEM as periodic homogenization technique consists of taking advantage of the periodicity of the physical properties of the piezocomposite by reducing the studied domain to a representative volume element (RVE) that should faithfully emulate the behavior of the entire piezocomposite. Here, the criterion for a good choice of the RVE is the equality in strain energy for the piezocomposite and homogenized structures. Figure 1(b) shows the finite element meshed model used in ANSYS for the RVE using a fiber volume fraction (FVF) of $50 \%$. The 3D 8-node coupled-field solid finite element SOLID5 was used to mesh all volumes. Here, 2580 elements and 3216 nodes were used. 


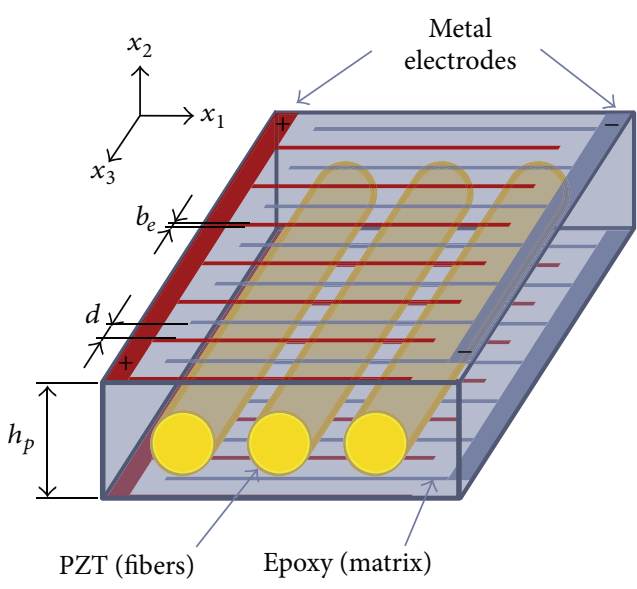

(a) Unidirectional (1-3 periodic) piezoelectric fiber composite

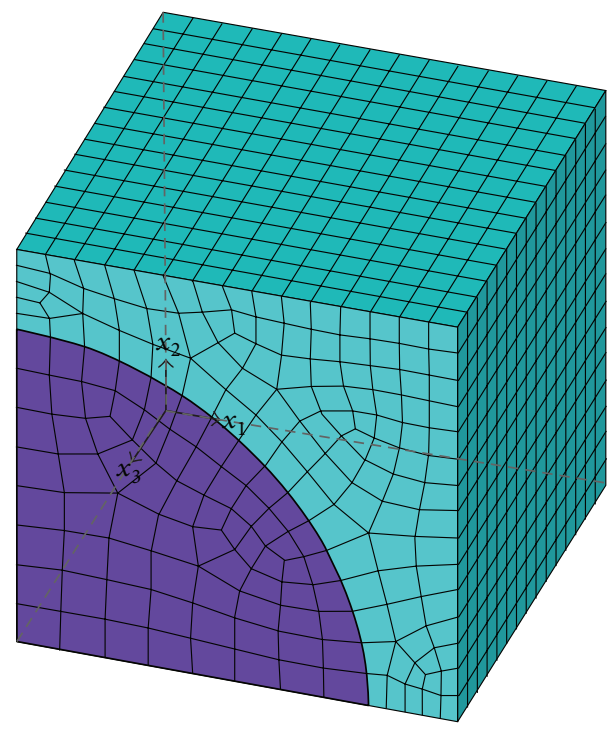

(b) $3 \mathrm{D}$ model of the RVE ( $50 \%$ of fiber)

FIGURE 1: Schematic of the active-fiber composite and its RVE.

TABLE 1: Material properties of the piezocomposite constituents: Fiber (PZT-5A) and Matrix (Epoxy) [23].

\begin{tabular}{lcccccc}
\hline & $C_{11}(\mathrm{GPa})$ & $C_{12}(\mathrm{GPa})$ & $C_{31}(\mathrm{GPa})$ & $C_{33}(\mathrm{GPa})$ & $C_{44}(\mathrm{GPa})$ & 21.05 \\
\hline PZT-5A & 120.35 & 75.18 & 75.1 & 110.87 & 9.48 & 0.86 \\
Epoxy & 9.48 & 7.75 & 7.75 & $e_{15}\left(\mathrm{C} / \mathrm{m}^{2}\right)$ & $\epsilon_{11}\left(10^{-9} \mathrm{~F} / \mathrm{m}\right)$ & $\epsilon_{33}\left(10^{-9} \mathrm{~F} / \mathrm{m}\right)$ \\
\hline & $e_{31}\left(\mathrm{C} / \mathrm{m}^{2}\right)$ & $e_{33}\left(\mathrm{C}^{2} \mathrm{~m}^{2}\right)$ & 12.3 & 9.16 & 8.30 \\
\hline PZT-5A & -5.35 & 15.78 & - & 0.885 & 0.885 \\
Epoxy & - & - & & & \\
\hline
\end{tabular}

Next, we calculate the effective electroelastic coefficients corresponding to tensile deformations of the chosen RVE, using ANSYS for different FVF [21]. To do so, the symmetry boundary conditions are firstly imposed on the planes $x_{1}=0$ and $x_{2}=0$. For a particular loading situation, only one value in the strain or electric field tensors is nonzero and all others become zero $[19,21]$.

In Figure 2, we plot the mechanical, electrical, and piezoelectric ratios between the effective values and the purely piezoelectric values, as a function of the FVF. We note that for high values of the FVF, the MTM is less relevant than FEM, because it assumes that the stress and strain fields inside the inclusions (fiber) are supposed to be constant, which is not the case of the FEM [19]. Thus, the FEM values corresponding to tensile deformations will be retained for the rest of this paper. However, since this FEM is not suitable to evaluate shear coefficients, where it results in a large overestimation [22], these coefficients $\left(e_{15}, C_{44}\right.$ and $C_{66}$ ) will be only determined by the MTM. In fact, while the high aspect ratio $\mathrm{EH}$ at hand is excited in the bending mode, structural shear deformations are not expected to be pronounced [15]. These latter will be used for 3D FEM of the EH.

\section{Electric Field Considerations in the Homogenized AFC}

In the case of homogenized AFC with IDEs, the corresponding electric field forms a set of curved lines, in the $\left(x_{3}-x_{2}\right)$ plane, along the direction of the fiber. Previous studies have shown that the electric field direction within the RVE is inhomogeneous and anisotropic [11]. In our case, for simplification purposes, the homogenized material is considered to be uniformly poled in the $x_{3}$-direction [10]. This simplification is adequate for large electrodes distances, as shown later.

An appropriate electrical representative volume element (eRVE) is chosen for the electric field analysis, in which we take advantage of the symmetry inside the AFC and the associated IDE. The eRVE is composed of a monolithic homogenized material with $50 \%$ of FVF. The corresponding material properties are shown in Table 2 where the material properties corresponding to shear deformation are computed using the MTM. The electrodes were not modeled explicitly, but voltages were applied directly on nodes located at the surface where electrodes are patterned. The symmetry boundary conditions are imposed on the eRVE at the planes $x_{1}=0$ and 

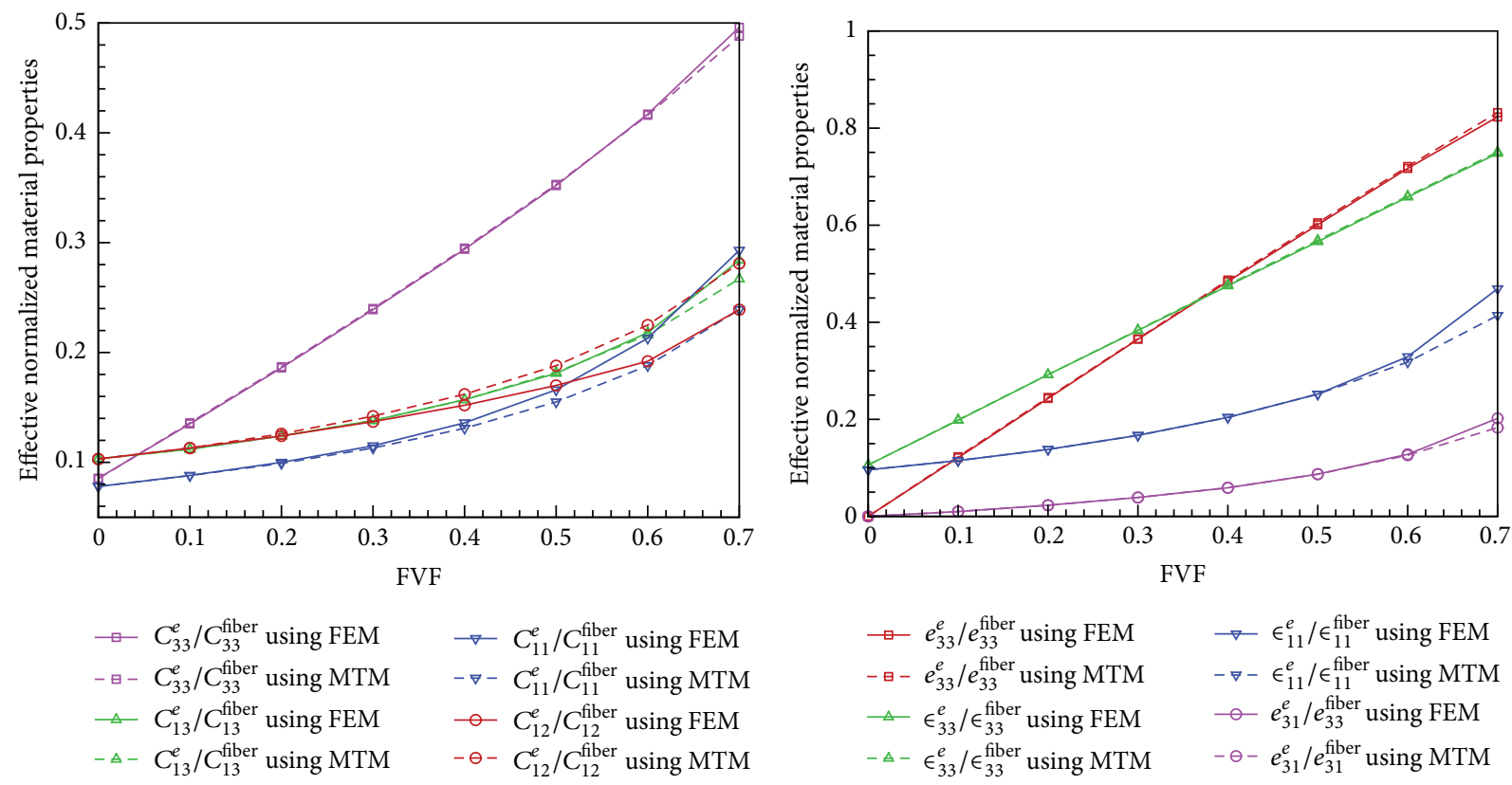

$\begin{array}{ll}-C_{33}^{e} / C_{33}^{\text {fiber }} \text { using FEM } & -\nabla-C_{11}^{e} / C_{11}^{\text {fiber }} \text { using FEM } \\ -\Xi-C_{33}^{e} / C_{33}^{\text {fiber }} \text { using MTM } & -\nabla-C_{11}^{e} / C_{11}^{\text {fiber }} \text { using MTM } \\ -\triangle C_{13}^{e} / C_{13}^{\text {fiber }} \text { using FEM } & --C_{12}^{e} / C_{12}^{\text {fiber }} \text { using FEM } \\ -\unlhd-C_{13}^{e} / C_{13}^{\text {fiber }} \text { using MTM } & -\ominus-C_{12}^{e} / C_{12}^{\text {fiber }} \text { using MTM }\end{array}$

(a) Mechanical properties

(b) Piezoelectric and electrical properties

FIGURE 2: Material mechanical, piezoelectric, and electrical properties of the piezocomposite as a function of the fiber volume fraction.

TABLE 2: Material properties of the homogenized material with $50 \%$ of fiber.

\begin{tabular}{|c|c|c|c|c|c|}
\hline$\overline{C_{11}^{e}(\mathrm{GPa})}$ & $C_{12}^{e}(\mathrm{GPa})$ & $C_{31}^{e}(\mathrm{GPa})$ & $C_{33}^{e}(\mathrm{GPa})$ & $C_{44}^{e}(\mathrm{GPa})$ & $C_{66}^{e}(\mathrm{GPa})$ \\
\hline 19.97 & 12.77 & 13.59 & 39 & 2.42 & 3.8 \\
\hline$\overline{e_{31}^{e}}\left(\mathrm{C} / \mathrm{m}^{2}\right)$ & $e_{33}^{e}\left(\mathrm{C} / \mathrm{m}^{2}\right)$ & $e_{15}^{e}\left(\mathrm{C} / \mathrm{m}^{2}\right)$ & $\epsilon_{11}^{e}\left(10^{-9} \mathrm{~F} / \mathrm{m}\right)$ & $\epsilon_{33}^{e}\left(10^{-9} \mathrm{~F} / \mathrm{m}\right)$ & \\
\hline-0.465 & 9.485 & 0.172 & 2.33 & 4.69 & \\
\hline
\end{tabular}

$x_{2}=0$ (Figure 6) and coupled displacements are applied on plane $x_{3}=0$ of the eRVE. The eRVE is meshed using 64256 nodes and 56250 elements with a 3D coupled-field element SOLID5 (Figure 3(a)).

For modeling purposes, we consider that the electric field is significant and homogeneous in the $x_{3}$ direction only. To justify this hypothesis, we study the effect of electrode separation $d$ for a range of electrode width $b_{e}$ on the fieldelongation coefficient $\alpha$ that characterizes the electric field shape's curvature in the $x_{3}$ direction

$$
\alpha=\frac{\left|\bar{E}_{3}\right|}{\left|\bar{E}_{2}\right|+\left|\bar{E}_{3}\right|} .
$$

In Figure 3(b), we can note that as the electrode separation is increased, $\alpha$ increases significantly. For large values of electrode separation $d$, the electric field becomes globally unidirectional and uniform along the $x_{3}$-axis. Thus, we can model the homogenized AFC as a piezoelectric material with transverse and parallel electrodes, in which the electrical field is uniform and aligned in the $x_{3}$-direction (Figure $5(\mathrm{a})$ ). This component is formed by a series of constant electric fields connected through the electrodes separated by the distance $d$ and electric potential $V$, that is,

$$
E_{3}=\frac{V}{d} h\left(x_{3}\right)\left(H\left(x_{2}\right)-H\left(x_{2}-h_{p}\right)\right),
$$

where $h\left(x_{3}\right)$ accounts for the spatial distribution of the electric field in the $x_{3}$-direction, and

$$
\begin{gathered}
h\left(x_{3}\right)=\sum_{i=1}^{(N-1) / 2}\left\{2 H\left(x_{3}-(2 i-1) d\right)-H\left(x_{3}-2 i d\right)\right. \\
\left.-H\left(x_{3}-(2 i-2) d\right)\right\} .
\end{gathered}
$$

Here $N$ is the number of electrodes (odd number), $H$ is the Heaviside step function, and $V(t)$ is the resulting potential difference between negative and positive electrodes.

\section{Energy Harvester Model and Analytical Solution}

We consider a bilayered cantilever beam in which one layer is made of aluminum and the other layer is made of an AFC piezocomposite patch partially covering the length of 


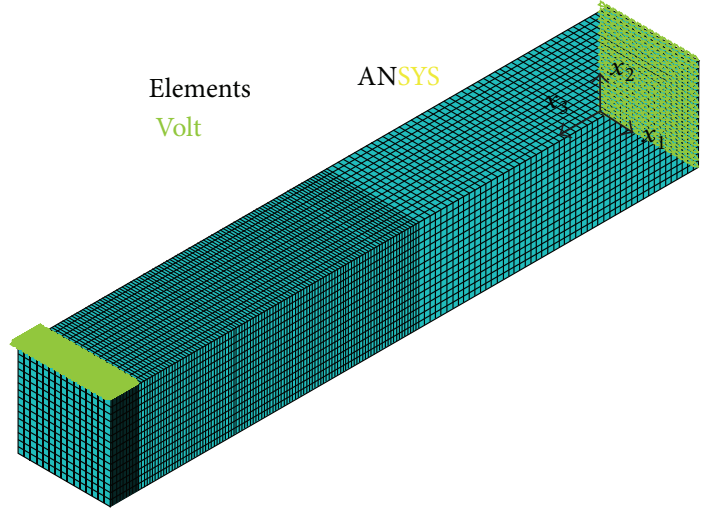

(a) Electrical representative elementary volume (eRVE)

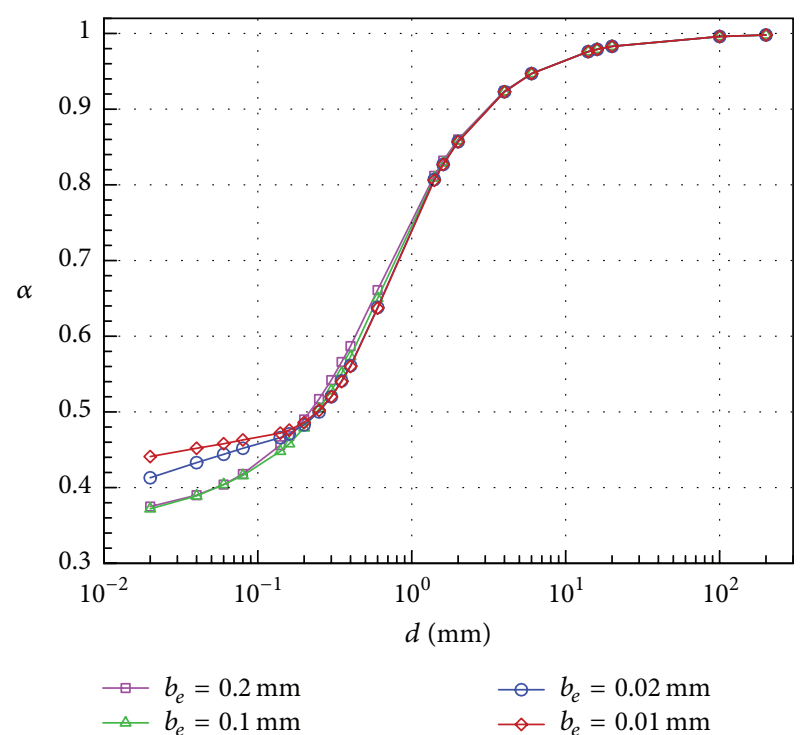

(b) Field-elongation coefficient $\alpha$ variation as function of electrode's separating distance $d$ for different values of electrode's width $b_{e}$

FIGURE 3: Electric field studies in the homogenized AFC.

the beam and placed near the clamped end. For modeling purposes, the piezocomposite is replaced by a homogenized material at $50 \%$ of FVF and crossed by $N$ transversal IDEs perpendicular to the $x_{3}$-direction, which corresponds to the $x$-axis of the beam, as shown in Figure 4(a). The clamped end of the beam is excited using a transverse harmonic displacement $Y(t)=Y_{0} \sin (\Omega t)$, where $\Omega$ is the driving frequency and $Y_{0}=0.1 \mathrm{~mm}$. In Table 3 , we give the material and geometrical properties of the bilayered cantilever beam, where the subscript $a$ refers to the aluminum layer and the subscript $p$ refers to the piezoelectric layer.

The constitutive equations of an isotropic material (aluminum layer) and transversely isotropic (piezoelectric layer) material are, respectively, represented by

$$
\begin{aligned}
& {\sigma_{11}}^{a}=E_{a}{ }^{*} \varepsilon_{11}=\frac{E_{a}}{1-v_{a}^{2}} \varepsilon_{11}, \\
& \sigma_{11}^{p}=E_{p}{ }^{*} \varepsilon_{11}-\bar{e}_{33}^{e} E_{1} \text {, }
\end{aligned}
$$

where $E_{p}{ }^{*}$ is the effective Young's modulus and $\bar{e}_{33}^{e}$ is the piezoelectric constant at the plane-stress assumption.

To analytically model our system, we start by assuming a beam that undergoes out-of-plane bending motion only characterized by the following displacement vector:

$$
\mathbf{R}=(v+Y) \mathbf{y}+y \mathbf{y}_{1},
$$

where $v(x, t)$ is the transverse displacement. $(\mathbf{x}, \mathbf{y}, \mathbf{z})$ is the inertial base frame, while the $\left(\mathbf{x}_{1}, \mathbf{y}_{1}, \mathbf{z}\right)$ is the local frame attached to the beam's cross-section obtained by a rotation with respect to the $z$-axis. Assuming small displacements, neglecting the rotary inertia, and applying Hamilton's principle, we use the Euler-Bernoulli beam theory to write the equation of motion

$$
\begin{gathered}
{\left[H_{1} E y_{3}+H_{2} E_{a}^{*} \frac{b}{3}\left(y_{1}^{3}-y_{0}^{3}\right)\right] v^{(4)}} \\
+\left[H_{1} e_{33}^{e} \frac{V(t)}{2 d} b h^{\prime \prime}(x)\left(y_{2}^{2}-y_{1}^{2}\right)\right] \\
+\left[H_{1} m_{1}+H_{2} m_{2}\right](\ddot{v}+\ddot{Y})=0,
\end{gathered}
$$

where $m_{1}=\rho_{a} b\left(y_{0}-y_{1}\right)+\rho_{p} b\left(y_{2}-y_{1}\right), m_{2}=\rho_{a} b h_{a}, E y_{3}=$ $b E_{a}{ }^{*} \int_{y_{0}}^{y_{1}} y^{2} d y+b E_{P}{ }^{*} \int_{y_{1}}^{y_{2}} y^{2} d y, H_{1}=H(x)-H\left(x-L_{1}\right)$, and $H_{2}=H\left(x-L_{1}\right)-H(x-L) \cdot y_{i}$ denotes the different layers positions (see Figure 4(b)).

To develop a reduced-order model of the system, we use the Galerkin procedure and let the displacement $v(x, t)$ be expressed as follows:

$$
v(x, t)=\sum_{r=1}^{\infty} \phi_{r}(x) \eta_{r}(t)
$$

where $\phi_{r}(x)$ is the $r$ th normalized mode shape of the free undamped eigenvalue problem associated with (9), when $L_{1}=L$ and $\eta_{r}(t)$ is the modal displacement [24].

Equation (10) is substituted into the equation of motion (9) and multiplied by the mode shape function; we use the orthogonality conditions and integrate over the beam's length. The coupled electromechanical ordinary differential equation of the modal response of the $\mathrm{EH}$ is obtained as

$$
M_{r} \ddot{\eta}_{r}(t)+C_{r} \dot{\eta}_{r}(t)+K_{r} \eta_{r}(t)+F_{r}=0,
$$




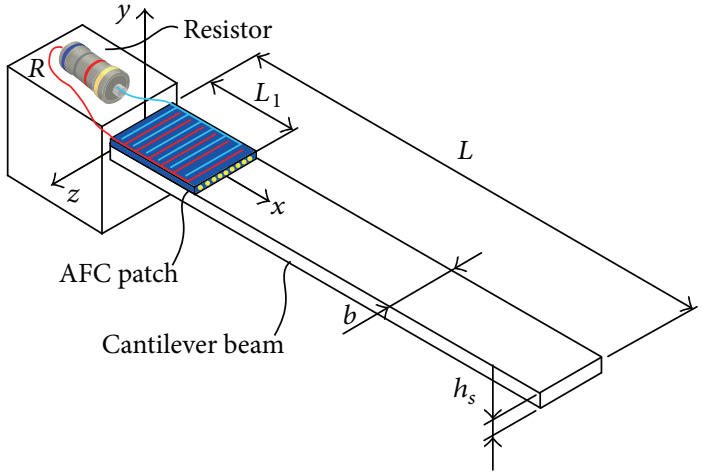

(a) Schematic of the bilayered cantilever beam

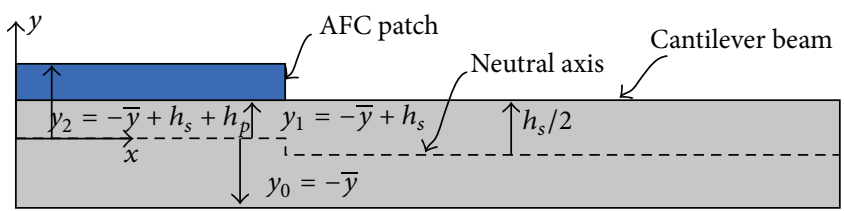

(b) Neutral axis position

FIGURE 4: Schematic of the energy harvester with its neutral axis position.

TABLE 3: Material and geometrical properties of the bi-layered cantilever beam.

\begin{tabular}{lcccccccc}
\hline$L(\mathrm{~mm})$ & $L_{1}(\mathrm{~mm})$ & $b(\mathrm{~mm})$ & $h_{p}(\mathrm{~mm})$ & $h_{a}(\mathrm{~mm})$ & $\rho_{p}\left(\mathrm{~kg} / \mathrm{m}^{3}\right)$ & $\rho_{a}\left(\mathrm{~kg} / \mathrm{m}^{3}\right)$ & $E_{a}(\mathrm{GPa})$ & $v_{a}$ \\
\hline 100 & 60 & 20 & 0.33 & 1 & 4475 & 2700 & 68 & 0.35 \\
\hline
\end{tabular}

where the modal mass term is

$$
M_{r}=\int_{0}^{L}\left[H_{1} m_{1}+H_{2} m_{2}\right] \phi_{r}^{2}(x) d x,
$$

the modal damping coefficient is

$$
C_{r}=2 \zeta_{r} \omega_{r} M_{r},
$$

where $\omega_{r}$ is the $r$ th natural frequency and $\zeta_{r}$ is the modal damping ratio $\left(\zeta_{1}=0.017\right)$, the modal mechanical stiffness term is

$$
K_{r}=\int_{0}^{L}\left[H_{1} E y_{3}+H_{2} E_{a} * \frac{b}{3}\left(y_{1}{ }^{3}-y_{0}{ }^{3}\right)\right] \beta_{r}{ }^{4} \phi_{r}{ }^{2}(x) d x,
$$

where $\beta_{r}$ is the $r$ th frequency number given in [24], and the modal electromechanical force is expressed as

$$
\begin{aligned}
F_{r}= & \int_{0}^{L} H_{1} \bar{e}_{33}^{e} \frac{b}{2 d}\left(y_{2}^{2}-y_{1}^{2}\right) h^{\prime \prime}(x) \phi_{r}(x) d x V(t) \\
& +\int_{0}^{L}\left[H_{1} m_{1}+H_{2} m_{2}\right] \phi_{r}(x) d x \ddot{Y}(t) .
\end{aligned}
$$

For the electrical modeling of the homogenized AFC, we use the simplification adopted in this paper when the electrical field is parallel to the $x$-axis. Therefore, we can model the AFC as a series of capacitors and current sources connected in parallel (Figure 5(b)).

The output voltage $V(t)$, also shown in Figure 5(b), represents the harvested voltage from the proposed $\mathrm{EH}$. This voltage is measured across the external resistor $R$ representing the external load. Applying Gauss's law on the whole piezoelectric patch yields

$$
\frac{d}{d t} \sum_{j=1}^{N-1} \int_{A_{j}} \mathbf{D}_{j} \cdot \mathbf{n}_{j} d A_{j}=\frac{V(t)}{R},
$$

where $\mathbf{D}_{j}$ is the electrical displacement vector between the electrodes, $\mathbf{n}_{j}$ is the electrodes normal vector, and the integration is performed over the electrode area $A_{j}$. Taking into account that the electrodes are parallel to the $(\mathbf{y}, \mathbf{z})$ plane and considering the homogenized piezoelectric constitutive equation, we obtain

$$
\mathbf{D}_{j} \cdot \mathbf{n}_{j}=D_{1}=\bar{e}_{33}^{e} \varepsilon_{11}-\bar{\epsilon}_{33}^{e} \frac{V(t)}{d},
$$

where $\bar{\epsilon}_{33}^{e}$ is the permittivity component at constant strain with the plane-stress assumption. Therefore, (15) is rewritten as

$$
\begin{aligned}
& (N-1) \bar{\epsilon}_{33}^{e} \frac{b}{d}\left(y_{1}-y_{2}\right) \dot{V}(t) \\
& \quad+\sum_{j=1}^{N-1} \int_{(j-1) d}^{j d} \bar{e}_{33}^{e}\left(y_{1}^{2}-y_{2}^{2}\right) \frac{b}{2 d} \dot{v}^{\prime \prime}(x, t) d x=\frac{V(t)}{R} .
\end{aligned}
$$

Applying Kirchhoff laws to the equivalent electrical circuit and using Galerkin decomposition, we obtain the following equation:

$$
C_{P} \dot{V}(t)+\frac{V(t)}{R}=i(t),
$$

where the internal equivalent capacitance term is expressed as

$$
C_{P}=(N-1) \bar{\epsilon}_{33}^{e} \frac{b}{d}\left(y_{2}-y_{1}\right),
$$

and the equivalent current source is expressed as

$$
i(t)=\sum_{r=1}^{\infty}\left(\left.\sum_{j=1}^{N-1} \bar{e}_{33}^{e}\left(y_{1}^{2}-y_{2}^{2}\right) \frac{b}{2 d} \frac{d \phi_{r}(x)}{d x}\right|_{x=(j-1) d} ^{x=j d}\right) \dot{\eta}_{r}(t) .
$$



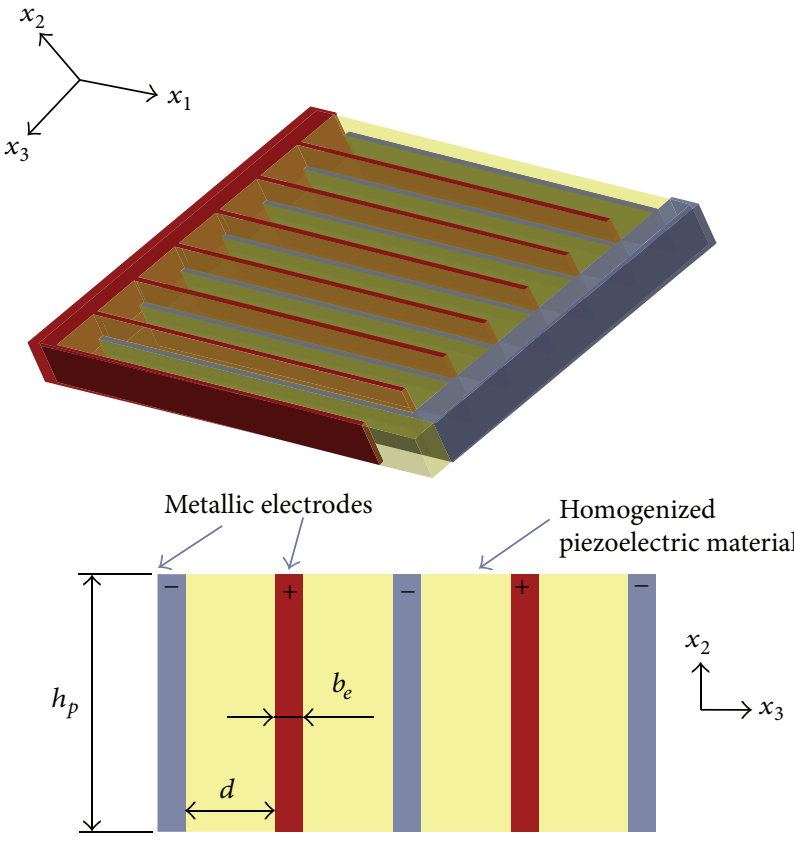

(a) Homogenized AFC patch

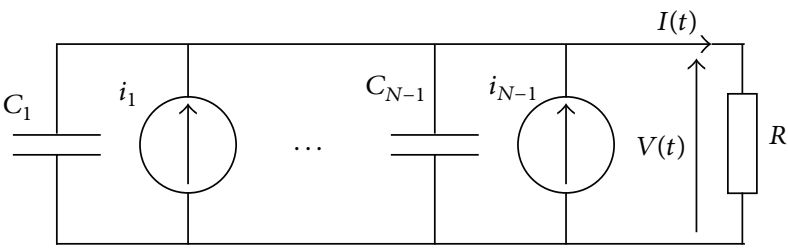

(b) Electrical model of the AFC

FIgURE 5: Modeling of the AFC patch.

Since the system is assumed to be linear, we assume that $\eta_{r}(t)=\operatorname{Im}\left(\eta_{r}^{0} e^{j \Omega t}\right)$ and $V(t)=\operatorname{Im}\left(V_{0} e^{j \Omega t}\right)$, where $\operatorname{Im}$ stands for the imaginary part, $j=\sqrt{-1}$ is the unit imaginary number, $\eta_{r}^{0}$ is the complex modal amplitude of the transverse displacement, and $V_{0}$ is the complex amplitude of the harmonic voltage across the resistive load. Solving the previous system analytically, we obtain the voltage $V_{0}$ amplitude as

$$
V_{0}=\frac{\sum_{r=1}^{\infty}\left(k_{r} m_{r} \Omega^{2} Y_{0} /\left(j \Omega C_{r}-M_{r} \Omega^{2}+K_{r}\right)\right)}{C_{p}-(j / \Omega R)+\sum_{r=1}^{\infty}\left(k_{r} f_{r} /\left(j \Omega C_{r}-M_{r} \Omega^{2}+K_{r}\right)\right)} .
$$

Assuming that excitation frequencies from the environment are usually low, the fundamental mode response will be dominant and constitute our main concern in this study $(r=$ 1).

\section{Typical Results and FE Validation}

5.1. FE Model. The FEM is used to validate the harvested voltage for different excitations near the fundamental frequency. To do so, a 3D FE model with 17 electrodes $(N=17$ and $\alpha=$ $0.92)$ is developed using ANSYS. Solid coupled field elements (SOLID5) are used for the piezoelectric behavior and circuit element (CIRCU94) for the piezoelectric-circuit analysis. A nil potential is applied at the nodes belonging to the negative electrodes. Also, we use equipotentiality condition for the positive electrodes' nodes. We then relate the resistive element $R$ to the electrodes through two active nodes located on the surfaces of negative and positive electrodes of the EH. A base displacement $Y_{0}$ is applied to the clamped side of the beam at frequency $\Omega$. The model, shown in Figure 6, has 22800 elements and 26313 nodes.

We start by calculating the mode shape and the natural frequencies of the system according to the FE model. The corresponding natural frequencies are shown in Table 4; they are compared to the ones obtained by the analytical model. As observed a mismatch of $10 \%$ is obtained. It is mainly due to the fact that the length of the AFC patch was taken to be equal to the aluminum beam in the analytical model.

5.2. Typical Results. Now, we study the response of the system to a harmonic excitation using the proposed analytical model, with one mode approximation in the Galerkin procedure, and compare the obtained results with those obtained using FE model at the same excitation conditions.

The results are given as a function of the normalized excitation frequency, obtained by dividing $\Omega$ by a factor so that the corresponding natural frequency is normalized to the FE short circuit natural frequency $\omega_{\mathrm{SC}}$, given in Table 4 .

We plot in Figure 7(a) the frequency response curves of the maximum output amplitude voltage $V_{\max }$ for different values of load resistance $R$, when the number of electrodes is $N=17$. In Figure 7(b), we vary the load resistance and look at the variation of $V_{\max }$ at the peak of the first resonant frequency; we denote this value by $V_{\max }^{\omega_{1}}$. As shown in Figure 7, a good agreement is obtained between analytical and FE simulations demonstrating the effectiveness of the proposed model. However, the proposed model is limited to an IDE EH with relatively large electrode's distance and very small electrode's width. The IDE EH performances could be improved by selecting the correct design parameters. Hence, 


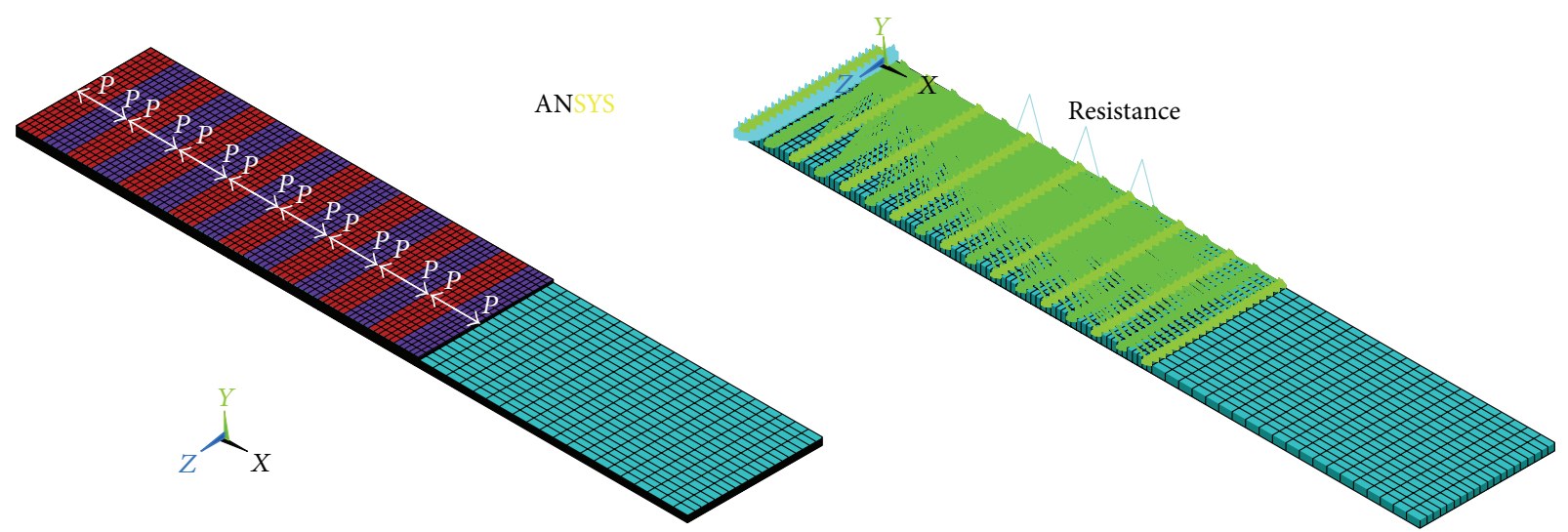

FIGURE 6: FE electromechanical model of the $\mathrm{EH}$ for $N=17$ electrodes (P: poling direction).

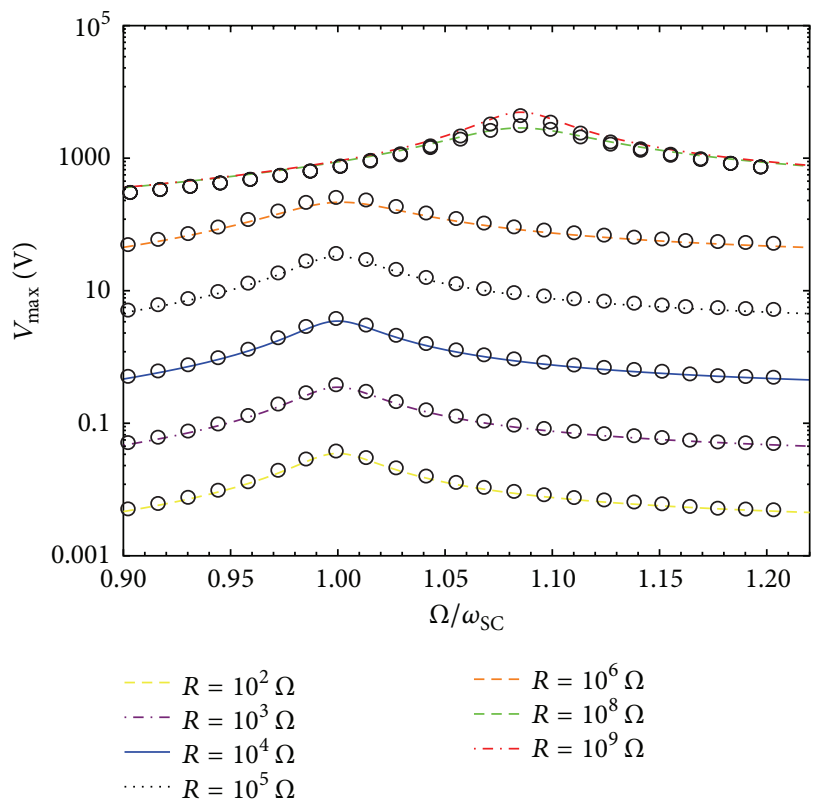

(a) Frequency response curves for $V_{\max }$ at different load resistances

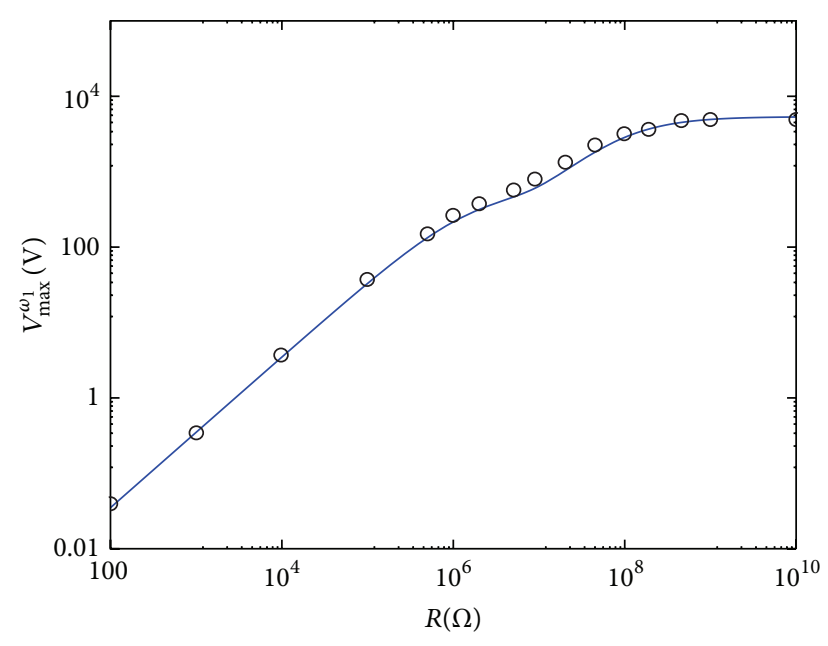

FIGURE 7: Analytical and FE harvested voltage at different excitation frequencies and load resistances: analytical prediction (continuous and dashed lines) and FE model (circles).

TABLE 4: Natural frequencies for open-circuit and short-circuit configurations when $N=17$ electrodes.

\begin{tabular}{lcc}
\hline & $\omega_{1} / 2 \Pi(\mathrm{FE})$ & $\omega_{1} / 2 \Pi$ \\
\hline Short-circuit frequency $(\mathrm{Hz})$ & 100.95 & 89.21 \\
Open-circuit frequency $(\mathrm{Hz})$ & 109.62 & 101.41 \\
\hline
\end{tabular}

the theoretically developed model can be used as basis for optimal design analysis.

\section{Conclusion}

In this paper, an analytical model of a vibrating structure, composed of an aluminum beam on top of which a piezoelectric composite patch, namely, AFC, has been attached, is derived and a closed-form solution is proposed. We use homogenization techniques to determine the effective properties of the piezoelectric composite, leading to a homogeneous model based on properties of the fiber and matrix constituents. We then used numerical simulations with ANSYS to extract the homogenized mechanical and electrical properties of the AFC. A simplified electrical field model is proposed in order to overcome the difficulty of resolving Gauss's equation for interdigitated-electrode configuration. We show that the proposed procedure could be applied to a complex piezoelectric microstructure in order to analytically derive and solve the equations of motion of such systems. We proposed closed-form solutions of the harvested electrical voltage. The proposed solutions were validated 
using finite element analysis for the same homogenized material. We demonstrated that the proposed solution is in good agreement with the finite element one even for critical load resistances.

\section{Conflict of Interests}

The authors declare that there is no conflict of interests regarding the publication of this paper.

\section{Acknowledgments}

A. Jemai, F. Najar, and M. Chafra are grateful for the funding provided to their laboratory by the Tunisian Ministry of Higher Education and Scientific Research. This work was performed under the framework of the NSF International Institute for Multifunctional Materials for Energy Conversion (NSF IIMEC); all the authors would like to acknowledge the financial support by NSF Grant no. DMR-0844082.

\section{References}

[1] A. Erturk and D. J. Inman, "An experimentally validated bimorph cantilever model for piezoelectric energy harvesting from base excitations," Smart Materials and Structures, vol. 18, no. 2, 18 pages, 2009.

[2] A. Abdelke, A. H. Nayfeh, M. R. Hajj, and F. Najar, "Energy harvesting from a multifrequency response of a tuned bendingtorsion system," Smart Materials and Structures, vol. 21, no. 7, Article ID 075029, 9 pages, 2012.

[3] J. H. Lim, C. H. Park, J. W. Kim, S. S. Jeong, M. H. Kim, and T. G. Park, "Generating characteristics of a cross-shaped piezoelectric generator depending on elastic body material and leg length," Journal of Electroceramics, vol. 30, no. 1-2, pp. 108112, 2013.

[4] H. A. Sodano, J. Lloyd, and D. J. Inman, "An experimental comparison between several active composite actuators for power generation," Smart Materials and Structures, vol. 15, no. 5, pp. 1211-1216, 2006.

[5] A. A. Bent, N. W. Hagood, and J. P. Rodgers, "Anisotropic actuation with piezoelectric fiber composites," Journal of Intelligent Material Systems and Structures, vol. 6, no. 3, pp. 338-349, 1995.

[6] R. B. Williams, D. J. Inman, M. R. Schultz, M. W. Hyer, and W. K. Wilkie, "Nonlinear tensile and shear behavior of macro fiber composite actuators," Journal of Composite Materials, vol. 38, no. 10, pp. 855-869, 2004.

[7] Q.-M. Wang, X.-H. Du, B. Xu, and L. Eric Cross, "Electromechanical coupling and output efficiency of piezoelectric bending actuators," IEEE Transactions on Ultrasonics, Ferroelectrics, and Frequency Control, vol. 46, no. 3, pp. 638-646, 1999.

[8] H. A. Sodano, G. Park, D. J. Leo, and D. J. Inman, "Use of piezoelectric energy harvesting devices for charging batteries," in Smart Structures and Materials 2003: Smart Sensor Technology and Measurement Systems, vol. 5050 of Proceedings of SPIE, 2003.

[9] L. J. Nelson, "Smart piezoelectric fibre composites," Materials Science and Technology, vol. 18, no. 11, pp. 1245-1256, 2002.

[10] C. R. Bowen, L. J. Nelson, R. Stevens, M. G. Cain, and M. Stewart, "Optimisation of interdigitated electrodes for piezoelectric actuators and active fibre composites," Journal of Electroceramics, vol. 16, no. 4, pp. 263-269, 2006.
[11] O. J. Myers, M. Anjanappa, and C. B. Freidhoff, "Numerical modeling of a circularly interdigitated piezoelectric microactuator," Journal of Microelectromechanical Systems, vol. 19, no. 5, pp. 1098-1104, 2010.

[12] M. W. Den Otter, "Approximate expressions for the capacitance and electrostatic potential of interdigitated electrodes," Sensors and Actuators A, vol. 96, no. 2-3, pp. 140-144, 2002.

[13] J. M. Lloyd, Electrical properties of macro-fiber composite actuators and sensors [M.S. thesis], Virginia Tech, 2004.

[14] S. Schaur and B. Jakoby, "An efficient method for modeling planar interdigitated electrodes for capacitive sensing," IEEE Sensors, vol. 13, pp. 1970-1973, 2011.

[15] A. Erturk, O. Bilgen, M. Fontenille, and D. J. Inman, "Piezoelectric energy harvesting from macro-fiber composites with an application to morphing-wing aircrafts," in Proceedings of the 19th International Conference on Adaptive Structures and Technologies, Ascona, Switzerland, October 2008.

[16] C. Mo, S. Kim, and W. W. Clark, "Theoretical analysis of energy harvesting performance for unimorph piezoelectric benders with interdigitated electrodes," Smart Materials and Structures, vol. 18, no. 5, Article ID 055017, 8 pages, 2009.

[17] R. R. Knight, C. Mo, and W. W. Clark, "MEMS interdigitated electrode pattern optimization for a unimorph piezoelectric beam," Journal of Electroceramics, vol. 26, no. 1-4, pp. 14-22, 2011.

[18] G. M. Odegard, "Constitutive modeling of piezoelectric polymer composites," Acta Materialia, vol. 52, no. 18, pp. 5315-5330, 2004.

[19] M. H. Malakooti and H. A. Sodano, "Multi-inclusion modeling of multiphase piezoelectric composites," Composites B, vol. 74, pp. 181-189, 2013.

[20] N. Fakri, L. Azrar, and L. El Bakkali, "Electroelastic behavior modeling of piezoelectric composite materials containing spatially oriented reinforcements," International Journal of Solids and Structures, vol. 40, no. 2, pp. 361-384, 2003.

[21] H. Berger, S. Kari, U. Gabbert et al., "Unit cell models of piezoelectric fiber composites for numerical and analytical calculation of effective properties," Smart Materials and Structures, vol. 15, no. 2, pp. 451-458, 2006.

[22] A. Deraemaeker, S. Benelechi, A. Benjeddou, and A. Preumont, "Analytical and numerical computation of homogenized prperties of MFCs: application to a composite boom with MFC actuators and sensors," in Proceedings of the 3rd Thematic Conference on Smart Structures and Materials (ECCOMAS '07), Gdansk, Poland, 2007.

[23] E. Lenglet, A.-C. Hladky-Hennion, and J.-C. Debus, "Numerical homogenization techniques applied to piezoelectric composites," Journal of the Acoustical Society of America, vol. 113, no. 2, pp. 826-833, 2003.

[24] L. Meirovitch, Fundamentals of Vibrations, McGraw-Hill, New York, NY, USA, 2001. 

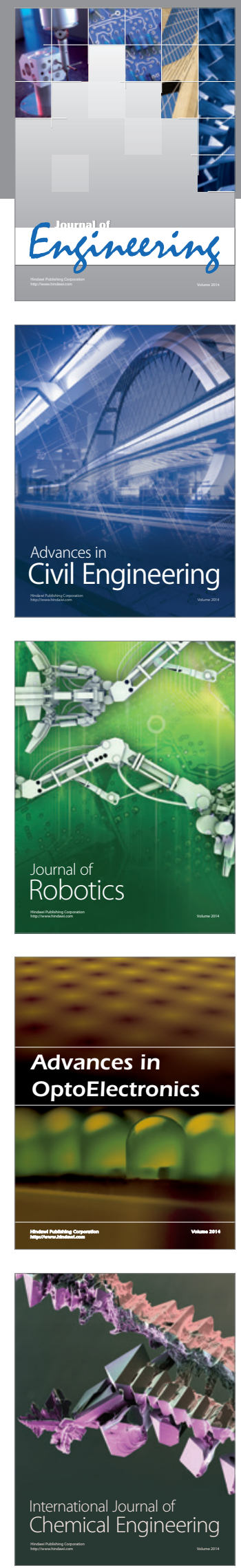

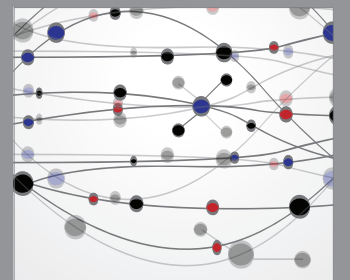

The Scientific World Journal
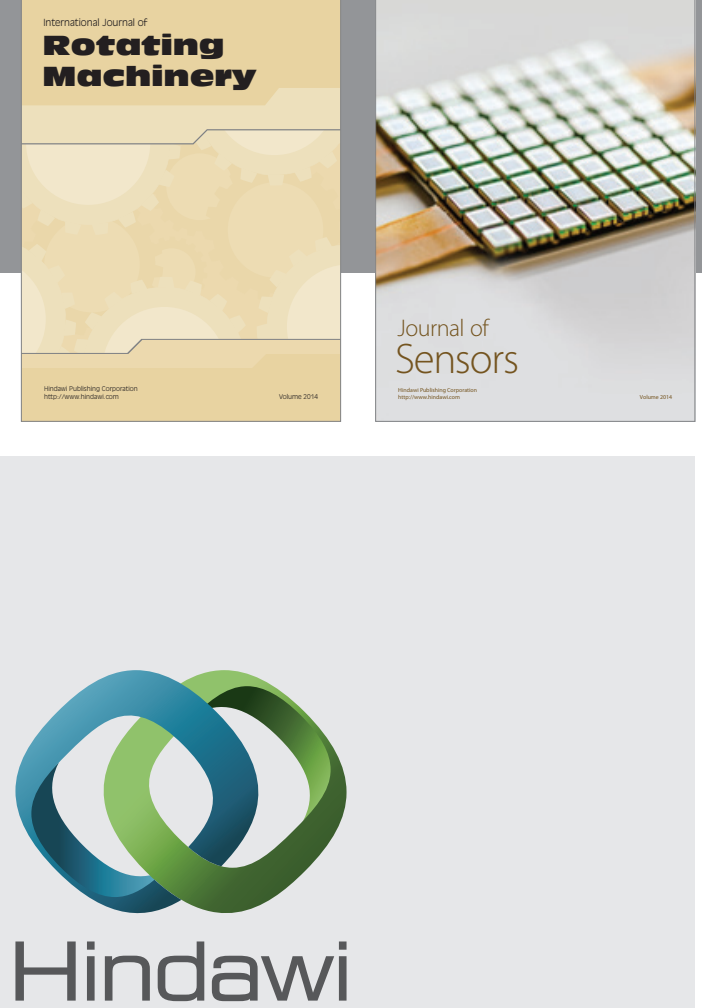

Submit your manuscripts at http://www.hindawi.com
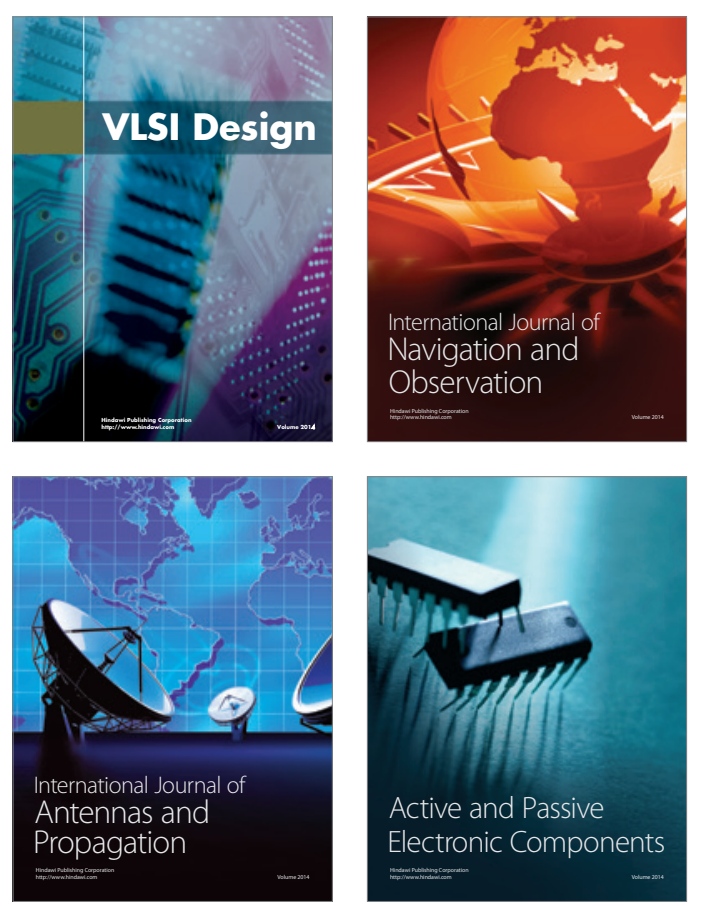
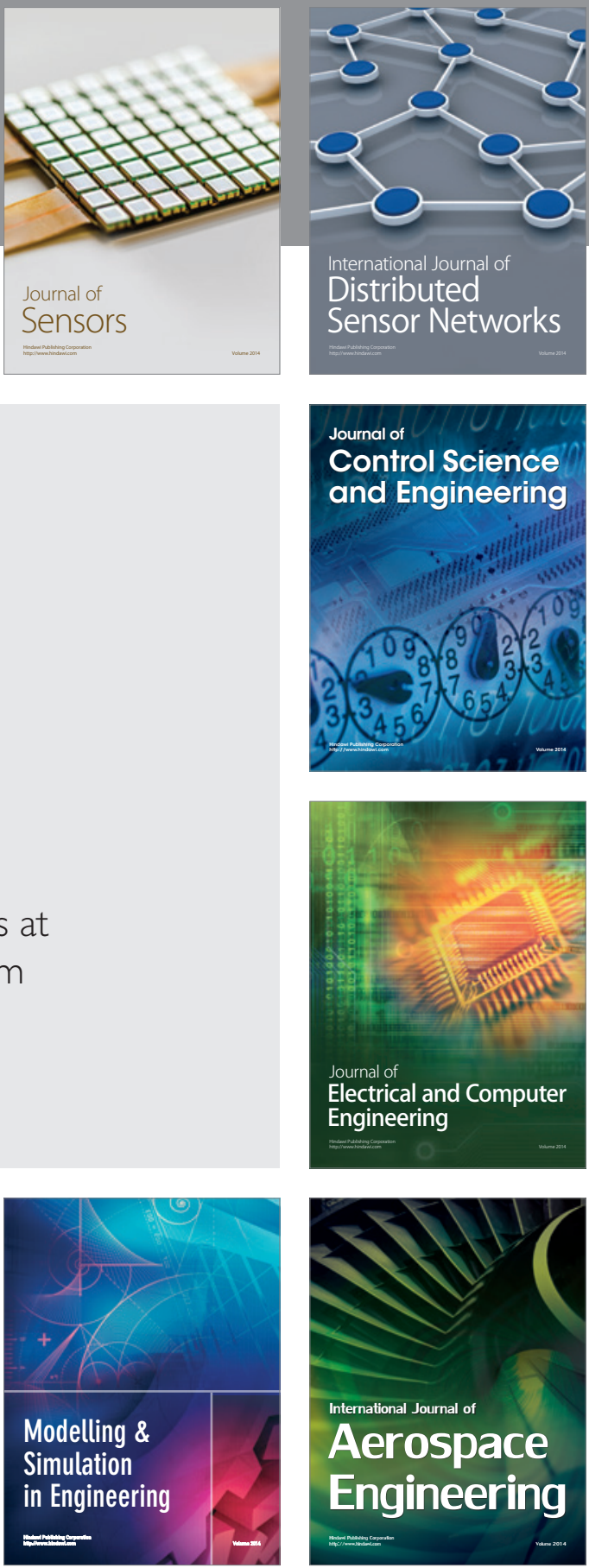

Journal of

Control Science

and Engineering
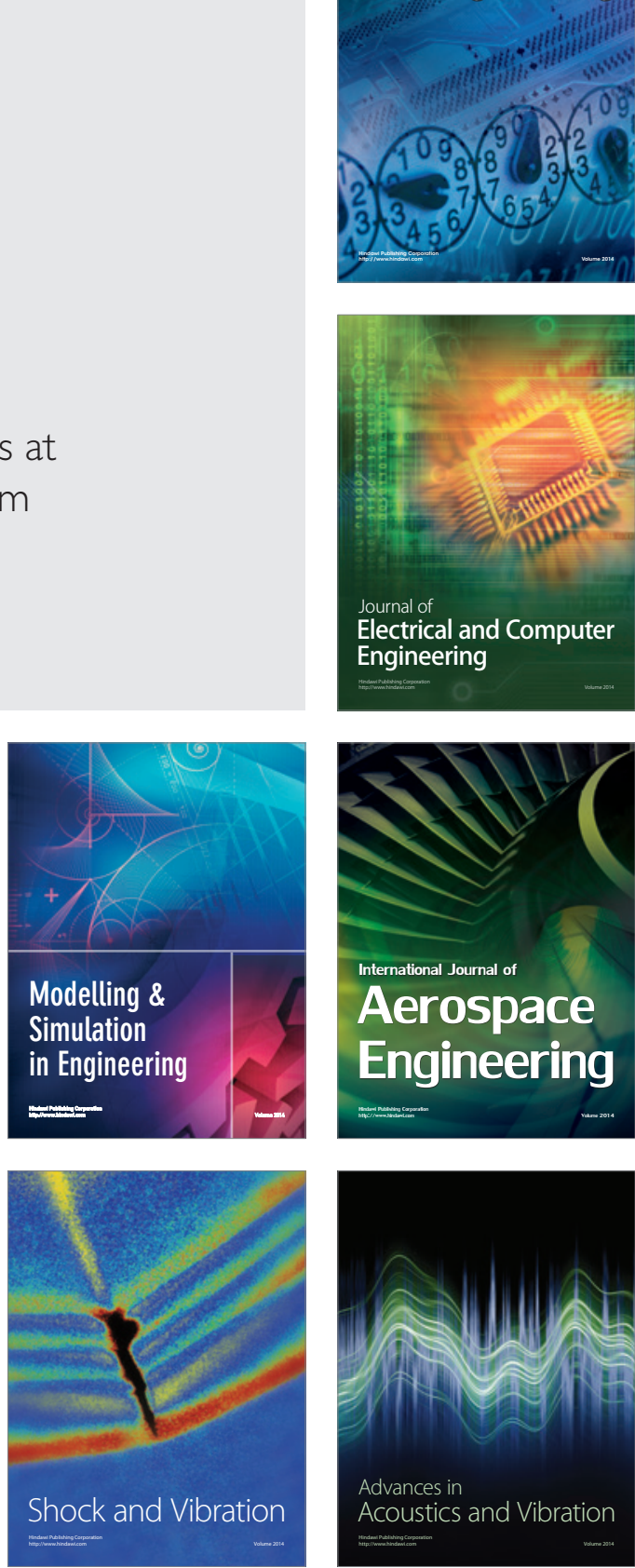